\title{
Polymer models of yeast $S$. cerevisiae genome organization
}

\author{
Geoff Fudenberg ${ }^{1 *}$, Jon-Matthew Belton², Anton Goloborodko ${ }^{3}$, Maxim Imakaev³, Job Dekker², Leonid Mirny ${ }^{3,4}$ \\ From Epigenetics \& Chromatin: Interactions and processes \\ Boston, MA, USA. 11-13 March 2013
}

\section{Introduction}

Three-dimensional (3D) chromosomal organization impacts critical cellular processes including transcription, replication, and genomic stability. Despite the ubiquity of these challenges, recent 3C-based experiments [1] suggest that major features of interphase chromosomal organization vary across eukaryotes. In human cells, $\mathrm{Hi}-\mathrm{C}$ revealed that chromosomal regions of similar functional state (eg. high gene expression) were enriched for contact probability in 3D [2]. Conversely, 3D contact probability was depleted between regions with different functional states. Moreover, the probability of a contact between two genomic loci was found to be inversely proportional to intervening genomic distance, $s^{1}$ and consistent with an unknotted non-equilibrium fractal globular polymer state. Here we analyze new, high-coverage, Hi-C data and developed stochastic simulations of polymer dynamics to study the spatial organization of yeast chromosomes.

\section{Results}

Unlike in human chromosomes, we find no evidence of a domain-type organization in yeast. Furthermore, we find that contact probability decays more like $s^{-3 / 2}$ with increasing genomic distance in cerevisiae; this indicates that yeast chromosomes do not exhibit a fractal globule organization. Instead, we find that a Rabl-like organization of chromosomes and constraints from the nucleolus appear to be the most prominent features of chromatin organization [3,4]. In this Rabl organization, centromeres are co-localized near the spindle pole body on one side of the nucleus. Our stochastic polymer simulations allow us to reconstruct conformational ensembles consistent with Hi-C maps. We demonstrate that a Rabl organization leads to cross-like patterns of interactions between centromeric regions, as observed in yeast $\mathrm{Hi}-\mathrm{C}$ data. Our simulations also allow us

${ }^{1}$ Biophysics, Harvard University, Boston, MA, 02115, USA

Full list of author information is available at the end of the article to match experimental data on diffusion of individual genomic loci; this allows us to study the temporal evolution of chromosomal conformations.

\section{Conclusions}

Our models show that: (1) yeast chromosomes are generally consistent with a mildly confined "equilibrium globular" polymer state, in contrast with observations in human cell lines; (2) tethering in a Rabl conformation induces a 'polymer brush' effect which reproduces the majority of observed intra- and inter- chromosomal Hi-C interactions in yeast; (3) rapid progression through the cell cycle allows for spatial, but not necessarily topological, equilibration of yeast chromosomes, limiting their mutual entanglement.

\section{Author details}

${ }^{1}$ Biophysics, Harvard University, Boston, MA, 02115, USA. ${ }^{2}$ Systems Biology, UMASS Medical School, Worcester, MA, 01605, USA. ${ }^{3}$ Physics, MIT, Cambridge, MA, 02139, USA. ${ }^{4}$ Division of Health Science and Technology, Harvard-MIT, Cambridge, MA, 02139, USA.

Published: 8 April 2013

\section{References}

1. Dekker J, Rippe K, Dekker M, Kleckner N: Capturing Chromosome Conformation. Science 2002, 295:1306.

2. Lieberman-Aiden E, van Berkum NL, Williams L, Imakaev M, Ragoczy T, Telling A, Amit I, Lajoie BR, Sabo PJ, Dorschner MO, Sandstrom R, Bernstein B, Bender MA, Groudine M, Gnirke A, Stamatoyannopoulos J, Mirny LA, Lander ES, Dekker J: Comprehensive Mapping of Long-Range Interactions Reveals Folding Principles of the Human Genome. Science 2009, 326:289.

3. Duan Z, Andronescu M, Schutz K, Mcllwain S, Kim YJ, Lee C, Shendure J, Fields S, Blau CA, Noble WS: A three-dimensional model of the yeast genome. Nature 2010, 465(7296):363-367.

4. Therizols P, Duong T, Dujon B, Zimmer C, Fabre E: Chromosome arm length and nuclear constraints determine the dynamic relationship of yeast subtelomeres. Proc Natl Acad Sci 2010, 107(5):2025-2030.

doi:10.1186/1756-8935-6-S1-P128

Cite this article as: Fudenberg et al:: Polymer models of yeast S. cerevisiae genome organization. Epigentics \& Chromatin 2013 6(Suppl 1):P128. 